\title{
Megapixel X-ray ghost imaging with a binned detector in the object arm
}

\author{
Haipeng Zhang (张海鹏) ${ }^{1,2,3}$, Ke Li (李 可) ${ }^{2}$, Feixiang Wang (王飞翔) $)^{2}$, Hong Yu (喻 虹 $)^{4}$, Changzhe Zhao (赵昌哲) $)^{1,2,3}$ ， \\ Guohao Du (杜国浩) ${ }^{2}$ ，Zhongliang Li (李中亮) ${ }^{2}$, Biao Deng (邓 彪) $)^{2}$, Honglan Xie (谢红兰) $)^{2}$, Shensheng Han (韩申生) $)^{4}$, \\ and Tiqiao Xiao (肖体乔) $1,2,3^{*}$ \\ ${ }^{1}$ Shanghai Institute of Applied Physics, Chinese Academy of Sciences, Shanghai 201800, China \\ ${ }^{2}$ Shanghai Synchrotron Radiation Facility/Zhangjiang Lab, Shanghai Advanced Research Institute, Chinese Academy of Sciences, Shanghai 201204, China \\ 3 University of Chinese Academy of Sciences, Beijing 100049, China \\ ${ }^{4}$ Shanghai Institute of Optics and Fine Mechanics, Chinese Academy of Sciences, Shanghai 201800, China
}

*Corresponding author: xiaotiqiao@zjlab.org.cn

Received September 16, 2021 | Accepted December 29, 2021 | Posted Online January 13, 2022

\begin{abstract}
At present, reconstruction of megapixel and high-fidelity images with few measurements is a major challenge for X-ray ghost imaging (XGI). The available strategies require massive measurements and reconstruct low-fidelity images of less than $300 \times 300$ pixels. Inspired by the concept of synthetic aperture radar, synthetic aperture XGI [SAXGI] integrated with compressive sensing is proposed to solve this problem with a binned detector in the object arm. Experimental results demonstrated that SAXGI can accurately reconstruct the $1200 \times 1200$ pixels image of a binary sample of tangled strands of tungsten fiber from 660 measurements. Accordingly, SAXGI is a promising solution for the practical application of XGI.
\end{abstract}

Keywords: X-ray ghost imaging; compressive sensing; megapixel imaging.

DOI: 10.3788/COL202220.033401

\section{Introduction}

Ghost imaging (GI), referred to as correlated imaging, is a nonlocalized imaging method that has attracted extensive attention over the past few decades ${ }^{[1-4]}$. Dependent on the flexible design of GI's dual beams, 'ghost' images can be obtained with spatial resolution beyond the Rayleigh diffraction limit ${ }^{[5]}$, even in poor illumination ${ }^{[6]}$ or turbulent environments ${ }^{[7]}$. As a result, GI has been investigated for application in various fields such as remote sensing $^{[8]}$, three-dimensional imaging ${ }^{[9]}$, and quantum lithography ${ }^{[10,11]}$. In principle, lensless $\mathrm{GI}^{[12-14]}$ is applicable to any wavelength, which has been demonstrated experimentally with $\mathrm{X}$-rays ${ }^{[1,2,15-20]}$, electrons ${ }^{[21]}$, atoms ${ }^{[22]}$, and neutrons ${ }^{[23]}$ in addition to visible ${ }^{[24,25]}$ and infrared light ${ }^{[26]}$.

Up to now, X-ray GI (XGI) has attracted extensive attention and has been investigated theoretically and experimentally in recent years with pseudo-thermal ${ }^{[1,15,17,20]}$, true-thermal ${ }^{[18]}$, and entangled X-ray sources ${ }^{[19]}$ and in many diverse strategies including real-space XGI ${ }^{[1,15,17,18,20]}$, Fourier-transform XGI ${ }^{[2]}$, phase contrast XGI ${ }^{[27]}$, and X-ray ghost tomography ${ }^{[16]}$. In 2016, Yu et al. experimentally demonstrated the Fourier-transform XGI method using X-rays of the Shanghai Synchrotron Radiation Facility (SSRF) and successfully reconstructed the amplitude and phase images of a multi-slit sample with an image size of
$300 \times 300$ pixels from 284 measurements ${ }^{[2]}$. Different from Fourier-transform XGI, in which a space-resolved detector is needed in the object arm, real-space XGI uses only a bucket detector, which has much higher sensitivity than a pixel-array detector. Using a true-thermal X-ray source generated by the isolated electron bunches of the European Synchrotron Radiation Facility (ESRF), Pelliccia et al. achieved the real-space XGI of a copper wire with an image of $30 \times 20$ pixels using the ensemble average algorithm from 20,000 measurements ${ }^{[18]}$. Then, in 2017, Schori et al. also realized real-space XGI of slits through a rotating sheet of copy paper illuminated by a laboratory $\mathrm{X}$-ray source, in which a $10 \mu \mathrm{m}$-wide slit with $20 \times 20$ pixels was restored from 3600 measurements, and a $100 \mu \mathrm{m}$-wide slit with $40 \times 40$ pixels was reconstructed from 2400 measurements ${ }^{[20]}$. Many excellent works were published in 2018, making it a year of great significance for the development of XGI. Schori et al. experimentally demonstrated XGI with paired X-ray photons generated by parametric down conversion at Spring-8, which has the potential to allow the observation of quantum phenomena at X-ray wavelength ${ }^{[19]}$. Pelliccia et al. reconstructed a tungsten coil image of $260 \times 230$ pixels with higher signal-to-noise ratio (SNR) from 5000 measurements using the Landweber-iteration algorithm with a pseudo-thermal $\mathrm{X}$-ray source produced by glass powder ${ }^{[17]}$. By pre-recording a 
series of repeatable reference patterns, Zhang et al. successfully imaged both a metal stencil and a conch with an image size of less than $250 \times 250$ pixels using ensemble averaging over 10,000 measurements ${ }^{[1]}$. Kingston et al. demonstrated ghost tomography with an aluminum cylinder with two different holes, in which the projection image of $64 \times 64$ pixels was retrieved from 2000 measurements ${ }^{[16]}$. Ceddia et al. proposed an experimental scheme for phase contrast XGI and demonstrated it by numerical simulation ${ }^{[27]}$. Two years later, He et al. ${ }^{[15]}$ applied the scheme of computational GI to restore a structured metal sample of $64 \times 64$ pixels through a deep learning algorithm from 768 frames, in which speckle patterns were generated by a designed orthogonal Hadamard matrix instead of silicon carbide sandpaper. Up to now, the image size achieved by XGI has been limited to $300 \times$ 300 pixels or less, which prevents the practical application of XGI to high-fidelity and megapixel imaging in biomedicine and material science.

Reconstruction of megapixel and high-fidelity images with few measurements is a major challenge for XGI. As is well known, the number of measurements needed for image reconstruction with acceptable SNR is proportional to the number of pixels in the image. For the reconstruction of a megapixel image based on the classic ensemble average algorithm, typically many more measurements than the Nyquist-Shannon criterion are needed to achieve sufficient fidelity, which implies tedious data acquisition and long acquisition time. Reducing the number of measurements significantly is apparently the critical path for the realization of practical XGI. The compressed sensing algorithm (CSA) is proposed for the reconstruction of XGI, which can reduce the acquisition time dramatically ${ }^{[28-31]}$. Unfortunately, the computing power and memory space required by the CSA are exceptionally large ${ }^{[32]}$, which ultimately limits the number of pixels of the ghost image that can be reconstructed. Many other strategies have been developed to significantly improve fidelity with fewer measurements than traditional GI, such as differential $\mathrm{GI}^{[33]}$, iterative denoise of $\mathrm{GI}^{[34]}$, higher-order $\mathrm{GI}^{[35,36]}$, and pseudo-inverse $\mathrm{GI}^{[37,38]}$. However, all of these algorithms for high-fidelity reconstruction of XGI are helpless when dealing with megapixel images, which prevents its practical application to clinical diagnosis and microscopic investigation.

Inspired by the concept of synthetic aperture radar (SAR), a method called synthetic aperture XGI (SAXGI) is proposed in this study to reconstruct megapixel XGI images with high fidelity using few measurements.

\section{Principle}

\subsection{Theory for synthetic aperture X-ray ghost imaging}

The experimental setup for SAXGI is shown in Fig. 1. The pseudo-thermal X-ray source is artificially created by sandpaper sheets acting as masks illuminated by a collimated monochromatic X-ray beam. The stochastic intensity fluctuation at different sampling times is achieved by scanning the sandpaper

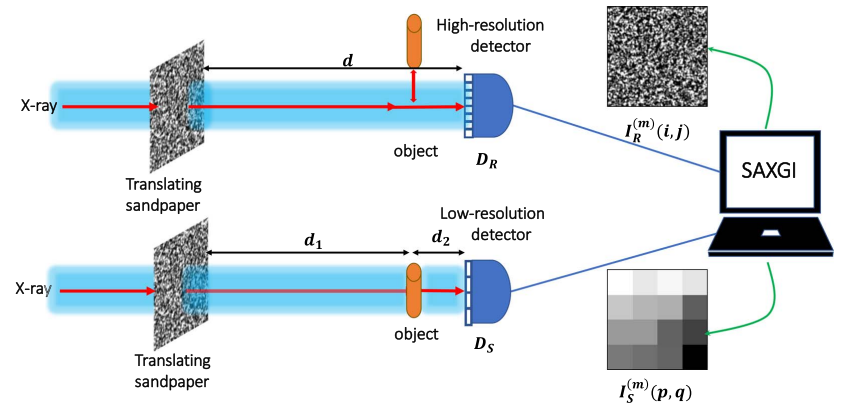

Fig. 1. Scheme of experimental setup for synthetic aperture X-ray ghost imaging (SAXGI), showing the reference arm and object arm.

transversely by a motor stage to illuminate different parts of the sandpaper. The object is placed a distance $d_{1}$ downstream of the sandpaper, followed by a high-resolution detector $D_{R}$ at a distance $d_{2}$ away from the object. Theoretically, the distance $d_{2}$ should be set to zero to meet the real-space GI condition of ensuring that the distance from pseudo-thermal source to object is equal to that from source to detector. However, a defocused distance is inevitable in actual experiments, and the increase of $d_{2}$ deteriorates the correlation strength between the speckle patterns on the object plane and detector plane in the reference arm and thus the quality of the reconstructed image. This implies that the object should be positioned as close as possible to the detector during data collection.

With the object out of the optical path (reference arm), the $\mathrm{X}$-ray beam propagates in free space and then impinges on $\mathrm{D}_{R}$. The chaotically distributed speckle pattern at the detector plane is registered by $D_{R}$. With the object moved back into the optical path (object arm), the X-ray beam penetrates through the object, and then the modulated X-ray beam is collected by a low-resolution detector $\mathrm{D}_{\mathrm{S}}$. The use of a low-resolution binned detector instead of a bucket detector in the object arm differentiates SAXGI from traditional XGI (TXGI). The pixels of $D_{S}$ are synthesized by binning over the adjacent pixels of $D_{R}$. The high-resolution reference patterns and the corresponding low-resolution signals are collected by shuttling the object in and out of the X-ray beam during the pseudo-coherent duration in each measurement (during which the pseudo-thermal X-ray source is relatively stable). At the $m$ th measurement, the speckle pattern in the reference arm and the low-resolution signal in the object arm are denoted as $I_{R}^{(m)}(i, j)$ and $I_{S}^{(m)}(p, q)$, respectively, where $(i, j)$ and $(p, q)$ indicate the pixel location on $D_{R}$ and $\mathrm{D}_{\mathrm{S}}$, respectively. After $M$ measurements, the structure information of the object could be retrieved according to the TXGI algorithm ${ }^{[17]}$ as follows:

$$
T_{\mathrm{TXGI}}=\langle(Y-\langle Y\rangle) A\rangle,
$$

where $\langle\cdot\rangle$ denotes ensemble averaging over all the measurements. According to Eq. (1), the reconstructed image by TXGI is synthesized through the linear superposition of the rows from the measurement matrix $A$. The weight coefficient of each 
row is the corresponding bucket signal $Y$ subtracted by its mean $\langle Y\rangle . A \in \mathbb{R}^{M \times N}$ comprises all the reference patterns,

$$
A=\left[\begin{array}{ccccc}
I_{R}^{(1)}(1,1) & \cdots & I_{R}^{(1)}(i, j) & \cdots & I_{R}^{(1)}\left(N_{1}, N_{2}\right) \\
\vdots & & \vdots & & \vdots \\
I_{R}^{(m)}(1,1) & \cdots & I_{R}^{(m)}(i, j) & \cdots & I_{R}^{(m)}\left(N_{1}, N_{2}\right) \\
\vdots & & \vdots & & \vdots \\
I_{R}^{(M)}(1,1) & \cdots & I_{R}^{(M)}(i, j) & \cdots & I_{R}^{(M)}\left(N_{1}, N_{2}\right)
\end{array}\right]_{M \times N},
$$

where $N=N_{1} \times N_{2}$ denotes the size of the high-resolution reference pattern taken by $D_{R}$ and the size of the target image T. $Y \in \mathbb{R}^{M \times 1}$ is regarded as an observation vector in TXGI,

$$
Y=\left[\begin{array}{c}
Y_{S}^{(1)} \\
\vdots \\
Y_{S}^{(m)} \\
\vdots \\
Y_{S}^{(M)}
\end{array}\right]_{M \times 1}
$$

where $Y_{S}^{(m)}=\sum_{p=1, q=1}^{s_{1}, s_{2}} I_{S}^{(m)}(p, q)$ signifies the sum over all the pixels of $\mathrm{D}_{\mathrm{S}}$ at the $m$ th measurement, and $s_{1} \times s_{2}$ indicates the size of the low-resolution signal taken by $\mathrm{D}_{\mathrm{S}}$.

To attain a good fidelity, TXGI based on ensemble averaging requires many more measurements than pixels of the reconstructed image. The large number of measurements makes TXGI unsuitable for practical applications concerning data acquisition efficiency. Thus, the concept of $\mathrm{SAR}^{[39]}$ is introduced into XGI to divide the target image into several non-overlapping blocks of $n_{1} \times n_{2}$ pixels. The overall sampling rate is $M /\left(N_{1} \times N_{2}\right)$ for TXGI, while the block sampling rate is $M /\left(n_{1} \times n_{2}\right)$ for SAXGI. In general, the size of the block image in SAXGI is much smaller than that of the whole image, and the overall sampling rate is several orders of magnitude lower than the block sampling rate, which implies that SAXGI is able to reconstruct X-ray images with very few measurements. During the reconstruction of each block, there is an obvious relation at each measurement, which is expressed as follows:

$$
I_{S}^{(m)}(p, q)=\sum_{\substack{i=(p-1) \times n_{1}+1, j=(q-1) \times n_{2}+1}}^{p \times n_{1}, q \times n_{2}} t(i, j) \cdot I_{R}^{(m)}(i, j)
$$

where $t(i, j)$ is the intensity transmittance on the pixel location $(i, j)$ of the target image $T$. Taking all measurements and noise into consideration, Eq. (4) can be rewritten in matrix form,

$$
\vec{I}_{S}^{p, q}=A^{p, q} \cdot \vec{T}^{p, q}+e_{n},
$$

where $e_{n}$ denotes environmental noise, and $\vec{I}_{S}^{p, q} \in \mathbb{R}^{M \times 1}$ is a column vector composed of a sequence of $I_{S}^{(m)}(p, q)$.
$\vec{T}^{p, q} \in \mathbb{R}^{n \times 1}$ is also a column vector rearranged from $T^{p, q}$, and $T^{p, q}$ comes from the rectangular area $\left[(p-1) \times n_{1}+1, p \times\right.$ $\left.n_{1}\right] \times\left[(q-1) \times n_{2}+1, q \times n_{2}\right]$ of the object's transmittance map T. $A^{p, q} \in \mathbb{R}^{M \times n}$ is a measurement matrix constituted of the same area of all the reference patterns, where $n=n_{1} \times n_{2}$ represents the size of the image block $T^{p, q}$.

The CSA is introduced to further reduce the number of measurements while retaining sufficient image quality. Combined with total variation (TV) regularization ${ }^{[40,41]}$, Eq. (5) is rewritten as

$$
T^{p, q}=\operatorname{argmin} \operatorname{TV}\left(T^{p, q}\right)+\lambda\left\|A^{p, q} \cdot \vec{T}^{p, q}-\vec{I}_{S}^{p, q}\right\|_{2}^{2},
$$

where $\lambda$ denotes the penalty parameter, and

$$
\operatorname{TV}\left(T^{p, q}\right)=\sum_{\substack{i=(p-1) \times n_{1}+1, j=(q-1) \times n_{2}+1}}^{p \times n_{1}, q \times n_{2}}|t(i+1, j)-t(i, j)|+|t(i, j+1)-t(i, j)| .
$$

As is well known, for a larger block, more measurements are required to maintain an acceptable block sampling rate and achieve a successful reconstruction of the block image. On the other hand, according to the principle of the CSA, an image block with small size generally leads to the loss of sparse characteristics. This means that the block size selected for SAXGI should be optimized. Simulations were carried out to investigate the effect of block size on the SAXGI image quality, and the results are presented in Section 2.2.

Finally, the large-size image is synthesized from a sequence of image blocks. The quality of the reconstructed images is evaluated by the structural similarity (SSIM) index ${ }^{[42]}$, which characterizes the similarity between the retrieved image and the target image. The theoretical maximum of the SSIM is one, which represents the perfect reconstruction. According to the principles, SAXGI is anticipated to simultaneously achieve megapixels and high fidelity under the premise of very few measurements.

\subsection{Effect of block size on the restored image}

When the block size is too small, it is difficult for the CSA to accurately describe the sparse characteristics of each block in natural images, which makes it invalid to exactly restore the images. Accordingly, the peak SNR (PSNR) of the restored images tends to decrease with reduced block size. A simulation was carried out to verify this property. In the simulation with $1 \%$ Gaussian white noise added, to achieve the efficient reconstruction of large images, the cameraman image with $300 \times 300$ pixels was selected as the natural image. Images were reconstructed at a series of block sizes. The appropriate number of speckle patterns was set according to the number of pixels in each block and the specific block sampling rate. The simulated speckle field was generated by the propagation of the light field modulated by the randomly distributed irregular particles in a 
sandpaper sheet. The sandpaper was produced by thousands of superpositions of the two-dimensional Gaussian distribution function with the fixed variance and the randomly set mean. The maximum value and full width at half-maximum (FWHM) of the second-order autocorrelation ${ }^{[43]}$ for all the speckle patterns were 1.4 and 5 pixels, respectively. Finally, natural images with different block sizes were reconstructed by augmented Lagrangian and alternating direction algorithms (TVAL3) ${ }^{[41]}$.

Simulation results for the effect of block size on the restored image at different sampling rates are shown in Fig. 2. Figure 2(a) presents the target image for comparison. Figure 2(b) corresponds to the square block size of 20. Due to the limited block size, deteriorated image quality can be observed directly, especially at the areas denoted with yellow and green boxes. With the block size increased to 40 , the corresponding image quality was visibly improved, as shown in Fig. 2(c). When the block size was 60, the improvement of image quality in Fig. 2(d) relative to that in Fig. 2(c) was not significant. To analyze the effect of block size on the image quality quantitatively, image reconstruction with a series of block sizes of SAXGI was carried out. As shown in Fig. 2(e), the PSNR of the restored images rises sharply with the block size until the block size reaches 20 . At a block size of 30 , the rising rate of the PSNR begins to decline. After a block size of 40, the increase of the PSNR tends to gentle. As shown in Fig. 2(e), for sampling rates of $20 \%, 30 \%$, and $40 \%$, the higher the sampling rate, the larger the PSNR. The trend of the PSNR with block size is similar for all measured sampling rates. By appropriately compromising the number of measurements and PSNR in actual SAXGI for radiology, a block size of 40 and sampling rate of $30 \%$ are generally preferred. To demonstrate the ability of SAXGI for megapixel imaging, a simulation using an image of SSRF scenery with $2048 \times 2048$ pixels was also carried out, and the detailed structure of the whole scenery was successfully restored as presented in Section 3.1.

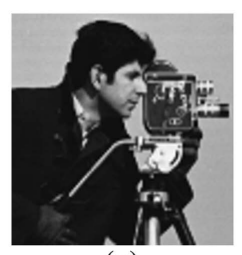

(a)

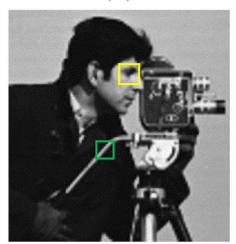

(c)

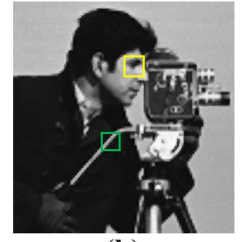

(b)

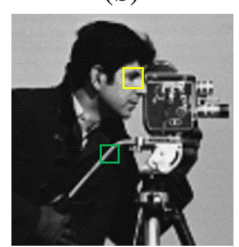

(d)

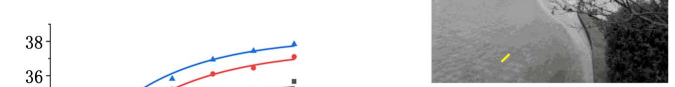

(a)

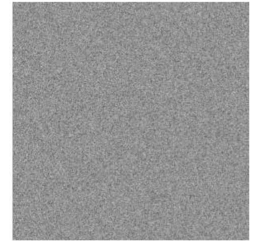

(c)

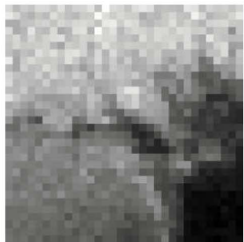

(b)

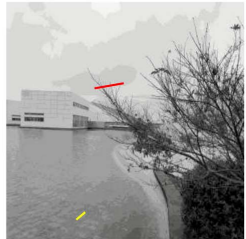

(d)

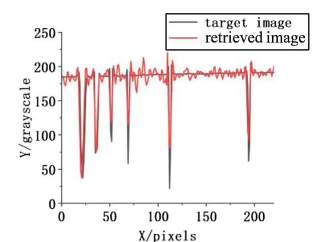

(e)

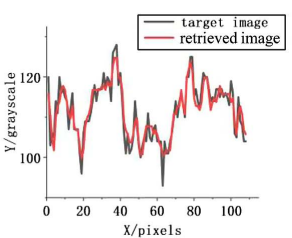

(f)
Fig. 3. Numerical simulation on the megapixel SAXGI. (a) Target image. (b) Signals in the object arm with binning of $64 \times 64$. (c) Image reconstructed by TXGI with 1000 measurements. (d) Image reconstructed by SAXGI with 1000 measurements. (e) Line profiles of the images marked at the red lines of (a) and (d). ( $f$ ) Line profiles of the images marked at the yellow lines of (a) and (d).

Fig. 2. Simulation results for the effect of block size on the restored images by SAXGI. (a) Target image. (b), (c), (d) Restored images at square block sizes of 20,40 , and 60, respectively, using a sampling rate of $30 \%$. (e) Peak signalto-noise ratio (PSNR) of the restored images versus block size at different sampling rates.

\section{Results and Discussion}

To demonstrate the ability of SAXGI for megapixel imaging, a the theoretical maximum of two, because the refractive index of the simulated speckle modulated the phase of X-rays within a range of much less than $2 \pi$, which is consistent with actual nditions.

tograph of the exterior scenery of SSRF with an image size of $2048 \times 2048$ pixels, as shown in Fig. 3(a). Shown in Fig. 3(b) was the signal recorded by the detector with $64 \times 64$ binning, which indicated that $n_{1}$ and $n_{2}$ were both set to 64 in the object arm. Figure 3(c) shows the image reconstructed by TXGI from lized by the CSA to ensure an effective image restoration is generally not lower than $30 \%$ of the Nyquist sampling limit ${ }^{[29-31,40]}$. In the case of a block sampling rate of $24.4 \%$, the corresponding overall sampling rate was $0.024 \%$, much lower than that required by the CSA, let alone all kinds of ensemble average (Therefore, it is reasonable that TXGI cannot achieve

Figure 3(d) shows the result of SAXGI, in which details of the target image, including the fuzzy branches of the bush and the of the CSA, which contributed to a high-fidelity and highresolution image restored by SAXGI. To compare the image

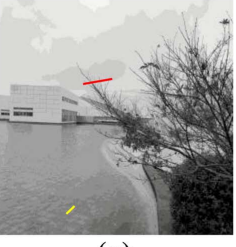


line profiles of the branches marked with red lines in Figs. 3(a) and 3(d) are presented in Fig. 3(e), which confirms the accurate reconstruction of the target image, especially for branches with larger size. Figure 3(f) shows the line profiles of ripples on the water surface of the pool marked with yellow lines in Figs. 3(a) and 3(d), respectively. Due to the low contrast induced by the ripples and reflection of the pool floor, the consistency of line profiles in Fig. 3(f) is poorer than that in Fig. 3(e). This demonstrates that objects with high contrast tend to be recovered with higher fidelity with the CSA based on TV regularization.

To further compare Figs. 3(d) and 3(a) quantitatively, the SSIM index is employed, and, in this case, its value is 0.982 , a value quite close to one, which indicates that the image reconstructed by SAXGI maintains high fidelity with the target image. Meanwhile, the SSIM value for the TXGI image is 0.009 , which agrees well with the fact that no effective spatial information of the target image can be retrieved through TXGI, as shown in Fig. 3(c). As verified by the numerical simulation, SAXGI is able to deal with megapixel image reconstruction with high fidelity under very limited measurements, while TXGI is ineffective under these conditions.

\subsection{Experiments}

A typical sample, especially a tangled strand of tungsten fiber, was chosen to test the feasibility of SAXGI for a binary object. The SAXGI experiments were carried out at beamlines of BL09B and BL13W at SSRF, a $3.5 \mathrm{GeV}$ third-generation synchrotron radiation facility ${ }^{[4]}$. The pseudo-thermal X-ray source was generated by scannable sandpaper scattered with irregular alumina particles, illuminated by a monochromatic X-ray beam at an energy of $15 \mathrm{keV}$. The average particle size of the sandpaper acting as the beam modulator is approximately $75 \mu \mathrm{m}$, which agrees with the FWHM of the second-order autocorrelation function ${ }^{[43]}$ of $72 \mu \mathrm{m}$ for all the reference patterns, as shown in Fig. 4(b). According to Fig. 4(b), the maximum of the autocorrelation function is approximately 1.26 . The larger the maximum value, the more intense the fluctuation of the speckle intensity, which results in a better measurement matrix to satisfy

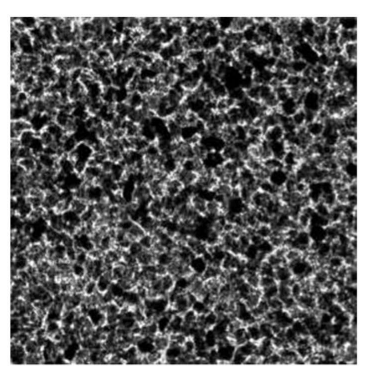

(a)

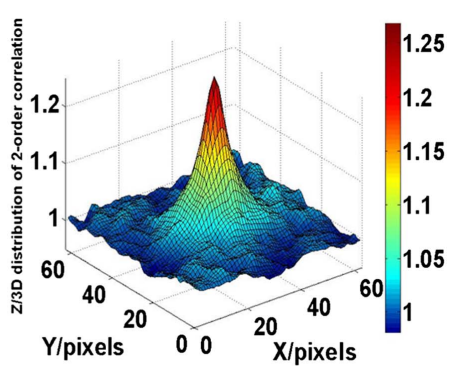

(b)
Fig. 4. Speckle pattern and corresponding autocorrelation function in the experiment. (a) Speckle pattern taken by the detector $D_{R}$ with the pixel size of $3.25 \mu \mathrm{m}$. (b) Autocorrelation function over the whole speckle pattern, which has the maximum value of 1.26 and full width at half-maximum (FWHM) of $72 \mu \mathrm{m}$.

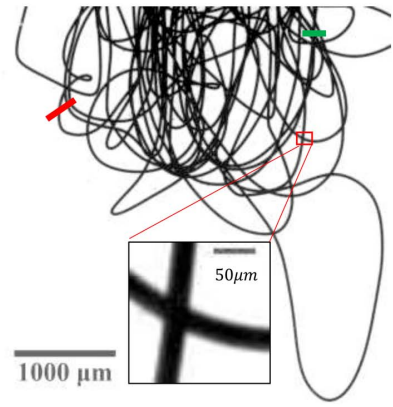

(a)

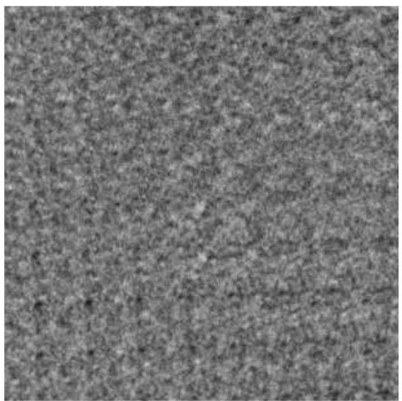

(c)

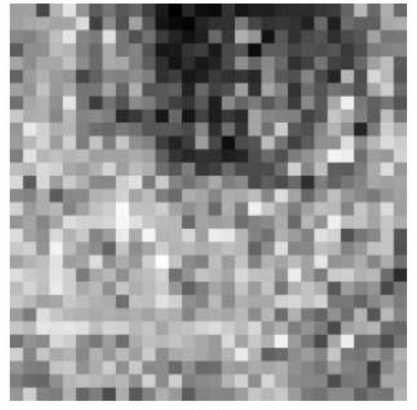

(b)

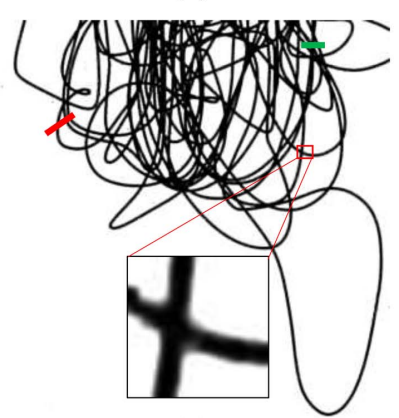

(d) the restricted isometry property (RIP ${ }^{[45,46]}$. Accordingly, XGI with the conditions mentioned above is noise resistant and able to restore an image with high fidelity from a few measurements. pere the Hamamatsu detector (model: ORCAobject. The basic pixel size of the Hamamatsu detector is $6.5 \mu \mathrm{m}$. The X-ray beam impinged on a scintillator to obtain a projected image of visible light and was then magnified with a microscope objective. The effective pixel size depends on the optical magnification. The decline of the correlation strength caused by the defocused distance $d_{2}<1 \mathrm{~cm}$ is inevitable in experiments. The introduced deterioration of image quality

expected to be acceptable in practical applications. contrast and complex structure for SAXGI. The target image shown in Fig. 5(a) was obtained by projection imaging with a object. In this experiment, after $2 \times$ optical magnification, the effective pixel size of $D_{R}$ is reduced to $3.25 \mu \mathrm{m}$, which is the basic pixel size divided by the optical magnification. Through a $40 \times 40$ binning of $D_{R}$, the effective pixel size of $D_{S}$ was $130 \mu \mathrm{m}$, which is much larger than the tungsten fiber's cross-sectional

Fig. 5. SAXGI experiments for tungsten fiber. (a) Target image taken by $D_{R_{r}}$ where the edge of the cross is smooth in the inset. (b) Signals in object arm with a binning of $40 \times 40$. (c) Image reconstructed by TXGI with 660 measurements. (d) Image reconstructed by SAXGI with the same measurements, where the edge of the cross is uneven in the inset. 
diameter of $20 \mu \mathrm{m}$. Figure 5(b) shows the object signals modulated by the sandpaper with a binning of $40 \times 40$. The exposure time for each frame in the signal and reference arm was increased to $6 \mathrm{~s}$, considering that the transmittance of the sandpaper is approximately $50 \%$. It is obvious that complex structure of the tungsten fiber cannot be revealed directly by the detector in the object arm, and correlation between signals and reference patterns is needed to retrieve the target image. A total number of 660 measurements were carried out during the data acquisition to implement the correlation. As shown in Fig. 5(c), it is obvious that TXGI is incapable of retrieving any structure information of the fiber at an overall sampling rate of $0.046 \%$.

Then, the reconstructed image by SAXGI is shown in Fig. 5(d). Obviously, the complex structure of the tungsten fiber was successfully retrieved, and the quality of the image restored by SAXGI was significantly improved compared with that by TXGI. Both the background noise and the complexity generated by the overlapping of the tungsten fiber may aggravate the difficulty of high-quality reconstruction. Therefore, the block sampling rate of $41.25 \%$ was employed to ensure accurate reconstruction for SAXGI. The similarity of the morphology of the crosses in the insets of Figs. 5(a) and 5(d) proved the reconstruction accuracy, although the edge of the tungsten fiber got slightly blurred in the reconstructed image. Results shown in Fig. 6 indicate that high consistency of the line profiles was achieved.

To evaluate the precision of the image reconstruction by SAXGI, line profiles of the sparse fibers denoted with red lines in Figs. 5(a) and 5(d) are presented in Fig. 6(a). High consistency between the SAXGI image and the target image can be observed, especially at positions with high contrast. As shown in Fig. 6(a), the flat top of the profile depicts the grayscale of the flat background without absorption. Moreover, line profiles of the stacked fibers denoted with green lines in Figs. 5(a) and 5(d) also match the positions and heights of the peaks of the curves quite well, as shown in Fig. 6(b). However, the consistency of the profiles at the lower and narrower peaks was obviously reduced, which confirms that simpler structure and higher contrast of objects would lead to higher fidelity of restoration.

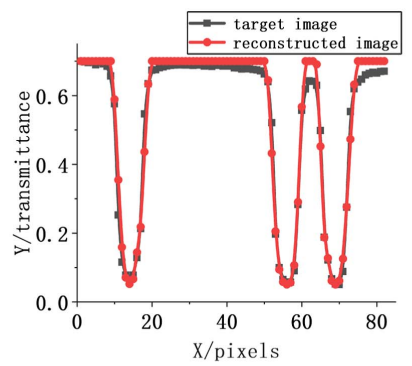

(a)

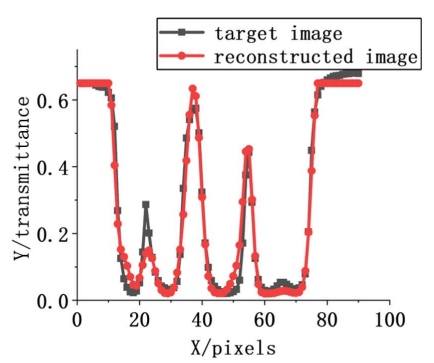

(b)
Fig. 6. Comparison between the line profiles of different positions in the target image and the reconstructed image. (a) Line profiles of the sparse tungsten fiber marked with red lines in Figs. 5(a) and $5(\mathrm{~d})$, respectively. (b) Line profiles of the stacked tungsten fiber marked with green lines in Figs. 5(a) and 5(d), respectively.
The SSIM value of 0.976 confirms the high fidelity of the tungsten fiber image reconstructed by SAXGI compared to the target image. Meanwhile, the SSIM value of 0.044 verifies the inability of TXGI to reconstruct the tungsten fiber from so few measurements.

Theoretically, the resolution limit of real-space XGI through the CSA can surpass the Rayleigh diffraction limit ${ }^{[15,29]}$. According to Fig. 4, the diffraction limit in the experiment is $72 \mu \mathrm{m}$, e.g., the FWHM of the autocorrelation function. Correspondingly, spatial resolution better than the diffraction limit can be achieved. However, the actual resolution is usually deteriorated by noises and insufficient sampling rate. As demonstrated in the experiments, the tungsten fibers with a diameter of $20 \mu \mathrm{m}$ were imaged distinctly, even though the two fibers were stacked very closely, which implies that spatial resolution of $20 \mu \mathrm{m}$ is achievable.

Image reconstruction efficiency is also important for applicability of the proposed method. The practical computing time for the reconstruction of ghost images depends on many factors including size of the block image, number of blocks, number of measurements, and image complexity. In the simulation, the time for the reconstruction of one block with a size of $64 \times 64$ pixels was $2.5 \mathrm{~s}$ for 1000 measurements, and the total reconstruction time for the megapixel image with total blocks of 1024 was $\sim 0.7 \mathrm{~h}$. In the experiments, the reconstruction time needed for a single block with size of $40 \times 40$ pixels was $1.6 \mathrm{~s}$ for 660 measurements. Accordingly, the final reconstruction time for the image of tungsten fibers composed of 900 blocks was about $\sim 0.4 \mathrm{~h}$, which is acceptable for a practical application of the proposed method. In general, the fewer measurements, the smaller block image, and the simpler image structure would decrease the time required for the image reconstruction of SAXGI. In principle, each block image can be reconstructed independently. Therefore, parallel computing based on a graphics processing unit (GPU) can be utilized to speed up significantly the reconstruction of the megapixel images.

As a result, experiments with a high absorption sample demonstrate the excellence of SAXGI for retrieving the image with high contrast and structure complexity from very few measurements.

\section{Conclusion}

As a powerful non-localized radiology, XGI has attracted extensive attention in recent years. However, reconstruction of megapixel and high-fidelity images with fewer measurements is a major challenge for XGI. Inspired by the concept of SAR, SAXGI is proposed in this study for the realization of megapixel and high-fidelity XGI using very few measurements. Both simulations and experiments were carried out to demonstrate the superiority of the proposed method. In the simulation, the SSIM up to 0.982 indicated that SAXGI almost perfectly retrieved the fine detail of the scenery image with $2048 \times 2048$ pixels from 1000 measurements. Experiments with a true binary sample of tangled strands of tungsten fiber verified that an image 
with $1200 \times 1200$ pixels was accurately reconstructed by SAXGI with $40 \times 40$ binning in the object arm from only 660 measurements, and its SSIM of 0.976 indicated that the complex structure of the tungsten fiber was restored successfully. Therefore, we can conclude that the proposed SAXGI is a promising solution for the implementation of megapixel and high-fidelity XGI with vastly fewer measurements than TXGI.

The ability of SAXGI for large field of view, high-fidelity XGI using few measurements implies that the proposed method may find applications in in-situ imaging of a variety of samples, especially when data acquisition efficiency is crucial. Moreover, when combined with computational XGI and a direct recording $\mathrm{X}$-ray detector in the signal arm, the data acquisition efficiency of SAXGI can be further improved, and a dynamic XGI is also predictable.

\section{Acknowledgement}

This work was supported by the National Key Research and Development Program of China (Nos. 2017YFA0206004, 2017YFA0206002, 2018YFC0206002, and 2017YFA0403801) and the National Natural Science Foundation of China (NSFC) (No. 81430087).

The authors thank Yanan Fu, Han Guo, Guanyun Peng, Yanling Xue, Fen Tao, and Zhijie Tan for their kind help and fruitful discussion on experiments and data processing.

\section{References}

1. A.-X. Zhang, Y.-H. He, L.-A. Wu, L.-M. Chen, and B.-B. Wang, "Tabletop X-ray ghost imaging with ultra-low radiation," Optica 5, 374 (2018).

2. H. Yu, R. Lu, S. Han, H. Xie, G. Du, T. Xiao, and D. Zhu, "Fourier-transform ghost imaging with hard X rays," Phys. Rev. Lett. 117, 113901 (2016).

3. Z. Bo, W. Gong, and S. Han, "Motion de-blurring by second-order intensitycorrelated imaging," Chin. Opt. Lett. 14, 070301 (2016).

4. A. Valencia, G. Scarcelli, M. D'Angelo, and Y. Shih, "Two-photon imaging with thermal light," Phys. Rev. Lett. 94, 063601 (2005).

5. J.-E. Oh, Y.-W. Cho, G. Scarcelli, and Y.-H. Kim, "Sub-Rayleigh imaging via speckle illumination," Opt. Lett. 38, 682 (2013).

6. W.-K. Yu, X.-F. Liu, X.-R. Yao, C. Wang, G.-J. Zhai, and Q. Zhao, "Singlephoton compressive imaging with some performance benefits over raster scanning," Phys. Lett. A 378, 3406 (2014).

7. J. Cheng, "Ghost imaging through turbulent atmosphere," Opt. Express 17, 7916 (2009).

8. C. Zhao, W. Gong, M. Chen, E. Li, H. Wang, X. Wendong, and A. Han, "Ghost imaging lidar via sparsity constraints," Appl. Phys. Lett. 101, 141123 (2012).

9. Y. Zhu, J. Shi, H. Li, and G. Zeng, "Three-dimensional ghost imaging based on periodic diffraction correlation imaging," Chin. Opt. Lett. 12, 071101 (2014).

10. A. N. Boto, P. Kok, D. S. Abrams, S. L. Braunstein, C. P. Williams, and J. P. Dowling, "Quantum interferometric optical lithography: exploiting entanglement to beat the diffraction limit," Phys. Rev. Lett. 85, 2733 (2000).

11. M. D'Angelo, M. V. Chekhova, and Y. Shih, "Two-photon diffraction and quantum lithography," Phys. Rev. Lett. 87, 013602 (2001).

12. X.-H. Chen, I. N. Agafonov, K.-H. Luo, Q. Liu, R. Xian, M. V. Chekhova, and L.-A. Wu, "High-visibility, high-order lensless ghost imaging with thermal light," Opt. Lett. 35, 1166 (2010).

13. X.-H. Chen, Q. Liu, K.-H. Luo, and L.-A. Wu, "Lensless ghost imaging with true thermal light," Opt. Lett. 34, 695 (2009).
14. M. Zhang, Q. Wei, X. Shen, Y. Liu, H. Liu, J. Cheng, and S. Han, "Lensless Fourier-transform ghost imaging with classical incoherent light," Phys. Rev. A 75, 021803 (2007).

15. Y.-H. He, A.-X. Zhang, M.-F. Li, Y.-Y. Huang, B.-G. Quan, D.-Z. Li, L.-A. $\mathrm{Wu}$, and L.-M. Chen, "High-resolution sub-sampling incoherent X-ray imaging with a single-pixel detector," APL Photonics 5, 056102 (2020).

16. A. M. Kingston, D. Pelliccia, A. Rack, M. P. Olbinado, Y. Cheng, G. R. Myers, and D. M. Paganin, "Ghost tomography," Optica 5, 1516 (2018).

17. D. Pelliccia, M. P. Olbinado, A. Rack, A. M. Kingston, G. R. Myers, and D. M. Paganin, "Towards a practical implementation of X-ray ghost imaging with synchrotron light," IUCrJ 5, 428 (2018).

18. D. Pelliccia, A. Rack, M. Scheel, V. Cantelli, and D. M. Paganin, "Experimental X-ray ghost imaging," Phys. Rev. Lett. 117, 113902 (2016).

19. A. Schori, D. Borodin, K. Tamasaku, and S. Shwartz, "Ghost imaging with paired X-ray photons," Phys. Rev. A 97, 063804 (2018).

20. A. Schori and S. Shwartz, "X-ray ghost imaging with a laboratory source," Opt. Express 25, 14822 (2017)

21. S. Li, F. Cropp, K. Kabra, T. J. Lane, G. Wetzstein, P. Musumeci, and D. Ratner, "Electron ghost imaging," Phys. Rev. Lett. 121, 114801 (2018).

22. M. Cao, X. Yang, J. Wang, S. Qiu, D. Wei, H. Gao, and F. Li, "Resolution enhancement of ghost imaging in atom vapor," Opt. Lett. 41, 5349 (2016).

23. A. M. Kingston, G. R. Myers, D. Pelliccia, F. Salvemini, J. J. Bevitt, U. Garbe, and D. M. Paganin, "Neutron ghost imaging," Phys. Rev. A 101, 053844 (2020).

24. T. Pittman, Y. Shih, D. Strekalov, and A. Sergienko, "Optical imaging by means of two-photon quantum entanglement," Phys. Rev. A 52, R3429 (1995).

25. D. Zhang, Y.-H. Zhai, L.-A. Wu, and X.-H. Chen, "Correlated two-photon imaging with true thermal light," Opt. Lett. 30, 2354 (2005).

26. H.-C. Liu and S. Zhang, "Computational ghost imaging of hot objects in long-wave infrared range," Appl. Phys. Lett. 111, 031110 (2017).

27. D. Ceddia and D. M. Paganin, "Random-matrix bases, ghost imaging, and X-ray phase contrast computational ghost imaging," Phys. Rev. A 97, 062119 (2018).

28. D. Donoho, "Compressed sensing," IEEE Trans. Inf. Theory 52, 1289 (2006).

29. W. Gong and S. Han, "High-resolution far-field ghost imaging via sparsity constraint," Sci. Rep. 5, 9280 (2015).

30. O. Katz, Y. Bromberg, and Y. Silberberg, "Compressive ghost imaging," Appl. Phys. Lett. 95, 131110 (2009).

31. G. Wu, T. Li, J. Li, B. Luo, and H. Guo, "Ghost imaging under low-rank constraint," Opt. Lett. 44, 4311 (2019).

32. R. Zhu, G.-S. Li, and Y. Guo, "Block-compressed-sensing-based reconstruction algorithm for ghost imaging," OSA Continuum 2, 2834 (2019).

33. F. Ferri, D. Magatti, L. A. Lugiato, and A. Gatti, "Differential ghost imaging," Phys. Rev. Lett. 104, 253603 (2010).

34. X.-R. Yao, W.-K. Yu, X.-F. Liu, L.-Z. Li, M.-F. Li, L.-A. Wu, and G.-J. Zhai, "Iterative denoising of ghost imaging," Opt. Express 22, 24268 (2014).

35. K. W. C. Chan, M. N. O'Sullivan, and R. W. Boyd, "High-order thermal ghost imaging," Opt. Lett. 34, 3343 (2009).

36. L.-H. Ou and L.-M. Kuang, "Ghost imaging with third-order correlated thermal light," J. Phys. B 40, 1833 (2007).

37. W. Gong, "High-resolution pseudo-inverse ghost imaging," Photonics Res. 3 , 234 (2015)

38. C. Zhang, S. Guo, J. Cao, J. Guan, and F. Gao, "Object reconstitution using pseudo-inverse for ghost imaging," Opt. Express 22, 30063 (2014).

39. W. M. Brown, "Synthetic aperture radar," IEEE Trans. Aerosp. Electron. Syst. AES-3, 217 (1967).

40. Y. Kang, Y.-P. Yao, Z.-H. Kang, L. Ma, and T.-Y. Zhang, "Performance analysis of compressive ghost imaging based on different signal reconstruction techniques," J. Opt. Soc. Am. A 32, 1063 (2015).

41. C. Li, An Efficient Algorithm for Total Variation Regularization with Applications to the Single Pixel Camera and Compressive Sensing (Rice University, 2011).

42. Z. Wang, A. Bovik, H. Sheikh, and E. Simoncelli, "Image quality assessment: from error visibility to structural similarity," IEEE Trans. Image Process. 13, $600(2004)$ 
43. X.-F. Liu, X.-R. Yao, M.-F. Li, W.-K. Yu, X.-H. Chen, Z.-B. Sun, L.-A. Wu, and G.-J. Zhai, "The role of intensity fluctuations in thermal ghost imaging," Acta Phys. Sin. 62, 184205 (2013).

44. H.-L. Xie, B. Deng, G.-H. Du, Y.-N. Fu, H. Guo, Y.-L. Xue, G.-Y. Peng, F. Tao, L. Zhang, and T.-Q. Xiao, "Methodology development and application of X-ray imaging beamline at SSRF," Nucl. Sci. Tech. 31, 102 (2020).
45. L. Pan, C. Deng, C. Yu, S. Yue, W. Gong, and S. Han, "Influence of the sparsity of random speckle illumination on ghost imaging in a noise environment," Chin. Opt. Lett. 19, 041103 (2021).

46. X. Xu, E. Li, X. Shen, and S. Han, "Optimization of speckle patterns in ghost imaging via sparse constraints by mutual coherence minimization," Chin. Opt. Lett. 13, 071101 (2015). 